\title{
Enhancing Treatment and Care of Children Using Pediatric Radiology
}

\author{
Manohar Shroff $^{1}$. Arun Kumar Gupta ${ }^{2}$
}

Received: 10 April 2019 / Accepted: 10 April 2019 / Published online: 26 April 2019

(C) Dr. K C Chaudhuri Foundation 2019

Radiology has become an indispensable tool in the evaluation of diseases of children. Pediatric radiology is a subspecialty which demands great attention to detail not only in the safe performance of the procedure but also in interpretation of acquired images. The Indian Journal of Pediatrics published three issues in June, July and September of 2016, dealing with modality based topics in Pediatric radiology. To follow up on the success of these issues, and to continue to keep updated on this rapidly changing and evolving field, IJP is again publishing symposia dedicated on pediatric radiology, in two parts. The first part dealt with bowel imaging, approach to neonatal jaundice, and MRI of the chest; and this second issue deals with genomics \& radiogenomics in inherited metabolic diseases, use and safety of gadolinium contrast in peditaric MR imaging, hydrocephalus in children, and imaging in short stature.

In the second issue, the first article "Genomics and Radiogenomics in Inherited Neurometabolic Disorders - A Practical Primer for Pediatricians" addresses the rapidly evolving field of radiogenomics to link genetics to clinical and radiologic phenotypes in inherited neurometabolic diseases [1]. In this article, Dr. Sniya Sudhakar and colleagues give an overview of the genetic basis of a disease, available genetic tests and the prominent role of radiology in contemplating a diagnostic suspicion and guiding further confirmatory tests. They have done a great job in simplifying the strategies for evaluation of genetic diseases and grouping them as, 1) disorders with clinical, imaging and genetic homogeneity, 2) disorders with similar clinical and imaging findings but involvement of different genes, and 3 ) disorders with different clinical and imaging findings due to involvement of the same

Manohar Shroff

manohar.shroff@sickkids.ca

1 Department of Diagnostic Imaging, Hospital for Sick Children/ Medical Imaging, University of Toronto, Toronto, Canada

2 Department of Radiodiagnosis, All India Institute of Medical Sciences, New Delhi, India gene. The article is exceptionally well illustrated and the authors have indeed truly created a practical primer on this subject.

The next article in this issue by Stephanie Holowka (MRI Safety Officer at SickKids, Toronto) and colleagues deal with the "Use and Safety of Gadolinium Based Contrast Agents in Pediatric MR Imaging" [2]. Gadolinium based contrast agents (GBCAs) have been used for over three decades with a high safety profile, however recent concerns regarding nephrogenic systemic fibrosis and gadolinium deposition have made it mandatory to rethink the risk-benefit analysis. New data has changed the approach on how to use GBCAs, with many institutions now developing policies on using such contrast agents only when really necessary and where it would make a difference to the diagnosis and further management. For Pediatricians ordering MRI with contrast, this is a must read.

The article on "Neuroimaging in Pediatric Hydrocephalus" by Dr. Pradeep Krishnan and colleagues addresses the common pediatric problem of hydrocephalus [3]. The authors of this article are well known for their expertise and experience in pediatric hydrocephalus. The role of recently discovered glial lymphatics and of aquaporin 4 receptors in CSF physiology has been addressed. They elegantly describe the pathophysiology of CSF secretion and hydrodynamics, and discuss the challenges with the old bulk flow theory and describe the newer hydrodynamic theory which divides hydrocephalus into acute and chronic or communicating hydrocephalus, a result of an increase in pulse pressure in brain capillaries and decreased intracranial compliance, which in turn maintains ventricular enlargement. The role of high resolution sequences like steady state T2 imaging to demonstrate exquisite anatomy for neurosurgical procedures like endoscopic third ventriculostomy is discussed and nicely illustrated. The authors round off the article with an interesting discussion on fetal hydrocephalus.

The final article in the second issue deals with the complex topic of "Imaging in Short Stature and Bone Age Estimation" [4]. Dr. Gupta and colleagues have provided us with an excellent review on the topic with clear, concise yet comprehensive 
approach to the problem of short stature and guidelines for imaging evaluation. In this article they have also addressed the paucity of data on bone age estimation in India, and have used their own extensive experience at AIIMS along with relevant literature to explain that the Tanner Whitehouse method (TW2) yields a more accurate result in Indian Children compared to the Greulich \& Pyle atlas method (GP), the caveat being that TW2 is more time intensive whereas the GP method is simple and quick. The article is also well illustrated with a suggested approach as an easy to follow flow diagram and examples of other causes of short stature such as pituitary abnormalities.

This symposium on Pediatric Radiology published in the IJP is an excellent addition to the symposium published in June, July and September 2016 issues of IJP. The articles are specifically written to educate and guide Pediatricians and Radiologists on the use of pediatric radiology to enhance treatment and care of children. These symposia on Pediatric Radiology will serve as a ready reckoner for both the practicing Pediatrician and the Radiologist.

\section{Compliance with ethical standards}

Conflict of Interest None.

\section{References}

1. Sudhakar SV, Muthusamy K, Arunachal G, Shroff M. Genomics and radiogenomics in inherited neurometabolic disorders - a practical primer for pediatricians. Indian J Pediatr. 2019. https:// doi.org/10.1007/s12098-019-02860-4.

2. Holowka S, Shroff M, Chavhan GB. Use and safety of gadolinium based contrast agents in pediatric MR imaging. Indian J Pediatr. 2019. https://doi.org/10.1007/s12098-019-02891-x.

3. Krishnan P, Raybaud C, Palasamudram S, Shroff M. Neuroimaging in pediatric hydrocephalus. Indian J Pediatr. 2019. https://doi.org/10. 1007/s12098-019-02962-z.

4. Gupta AK, Jana M, Kumar A. Imaging in short stature and bone age estimation. Indian J Pediatr. 2019. https://doi.org/10.1007/s12098019-02920-9.

Publisher's Note Springer Nature remains neutral with regard to jurisdictional claims in published maps and institutional affiliations. 